\title{
The effect of non-migrating tides on the morphology of the equatorial ionospheric anomaly: seasonal variability
}

\author{
Scott L. England ${ }^{1}$, Xiaoli Zhang ${ }^{2}$, Thomas J. Immel ${ }^{1}$, Jeffrey M. Forbes ${ }^{2}$, and Robert DeMajistre ${ }^{3}$ \\ ${ }^{1}$ Space Sciences Laboratory, University of California Berkeley, Berkeley, California 94720, USA \\ ${ }^{2}$ Department of Aerospace Engineering Sciences, University of Colorado, Boulder, Colorado 80309, USA \\ ${ }^{3}$ Applied Physics Laboratory, John Hopkins University, Laurel, Maryland, USA
}

(Received October 15, 2007; Revised May 30, 2008; Accepted July 3, 2008; Online published May 14, 2009)

\begin{abstract}
Recent observations of the low-latitude $F$-region ionosphere at times near equinox have shown that it varies with a predominant zonal wavenumber-four pattern in a fixed local-time frame. It has been shown that this pattern corresponds well to the non-migrating diurnal eastward wavenumber-three atmospheric tide (DE3) at $E$-region altitudes simulated by the Global Scale Wave Model (GSWM). Here we present details of the morphology of the $F$-region ionosphere from TIMED GUVI with simultaneous observations of the non-migrating diurnal tides at $E$-region altitudes from TIMED SABER. For the case of equinox (March 2002), the correspondence of the SABER and GUVI observations confirms the relationship previously established using the GSWM simulations. There is also a wavenumber-one signature that is present which may be related to the semi-diurnal westward wavenumber-three, possibly in conjunction with changes in the magnetic field with longitude. During July 2002, when the amplitude of the DE3 maximizes, the amplitude of the wavenumber-four pattern in the $F$ region ionosphere intensifies. There is also evidence of a strong wavenumber-three pattern in the $F$-region ionosphere, which can be attributed to the strong diurnal eastward wavenumber-two tide during this period. During January 2003, the amplitude of all non-migrating components observed by SABER are either small or asymmetric and the ionosphere does not display either a wavenumber-three or -four pattern. During both solstice periods, a strong wavenumber-one is seen that is attributed to the offset of the subsolar point and the geomagnetic equator that maximizes at solstice, possibly in conjunction with other geomagnetic effects. During all seasons, significant hemispheric asymmetries in the airglow wavenumber spectra are seen. The combined GUVI and SABER observations presented here demonstrate that the large-scale periodic longitudinal structure of the $F$ region ionosphere responds significantly to changes in the forcing by non-migrating diurnal tides at $E$-region altitudes.
\end{abstract}

Key words: Equatorial ionosphere, atmospheric tides, equatorial ionization anomaly.

\section{Introduction}

The plasma density in the low latitude ionosphere is most concentrated in two bands, each around $15^{\circ}$ from the magnetic equator (Namba and Maeda, 1939; Appleton, 1946). This feature is referred to as the equatorial ionospheric anomaly (EIA). It is created by the uplift of $F$-region plasma close to the magnetic equator, followed by the subsequent redistribution of that plasma to lower altitudes, following the magnetic field lines. This uplift is caused by polarization electric fields produced by a combination of tidal winds at $E$-region altitudes (Tarpley, 1970), which dominate at most local times (LT), and $F$-region winds, which dominate around sunset (Woodman, 1970; Rishbeth, 1971; Farley et al., 1986).

In the hours following sunset, the distribution of the $F$ region plasma density can be measured using airglow observations. Observations of the $135.6 \mathrm{~nm}$ OI emissions by IMAGE FUV and TIMED GUVI from around equinox have revealed a global scale with a zonal wavenumber-four

Copyright (c) The Society of Geomagnetism and Earth, Planetary and Space Sciences (SGEPSS); The Seismological Society of Japan; The Volcanological Society of Japan; The Geodetic Society of Japan; The Japanese Society for Planetary Sciences; TERRAPUB pattern in the brightness and magnetic latitude of the airglow arcs, when reconstructed at a fixed local time (around 21:00 LT) (Sagawa et al., 2005; Henderson et al., 2005b). Similar patterns have also been revealed in maps of the total electron content at equatorial latitudes (Lin et al., 2007; Scherliess et al., 2008). By comparing such observations to the results of the global scale wave model (GSWM), it has been argued that this pattern could be explained by a modulation of the $E$-region winds by the non-migrating diurnaleastward-wavenumber three tide (Immel et al., 2006; England et al., 2006a), that appears as a wavenumber-four pattern in a fixed-local-time reference frame. This is a result of the transformation from a fixed-universal-time reference frame in which the wavenumber of the tide is defined to the westward-propagating fixed-local-time reference frame in which the observations of the tide's effect are reported. England et al. (2006b) have shown that for these conditions, a similar wavenumber-four pattern is also seen in the noontime equatorial electrojet. This shows that a wavenumberfour modulation is indeed present in the dayside equatorial $E$-region which is consistent with tidal wind modulation of the polarization electric fields at this altitude. Similar modulations have also been observed in the vertical 
plasma drifts in the dayside $F$-region that result from these polarization fields (Hartman and Heelis, 2007; Kil et al., 2007). Kil et al. (2007) have shown that a wavenumber-four is not observed in the vertical drifts around sunset, when the $F$-region dynamo fields dominate. Hagan et al. (2007) have shown by forcing the lower boundary of the TIMEGCM model with a DE3 tide during equinox conditions, a wavenumber-four pattern in the $F$-region ion density can be produced, although some differences between the modeled and observed patterns were noted in this preliminary study. England et al. (2008) have shown that by modulating the horizontal electric field associated with the longitudinal variations observed by the CHAMP, Ørsted and SAC$\mathrm{C}$ satellites in the SAMI2 model, a change in the airglow comparable to that observed by TIMED-GUVI could be accounted for.

One thing that remains to be done is to compare the observations of the $F$-region plasma distributions with simultaneous observations of the tides at $E$-region altitudes. The TIMED satellite offers an ideal opportunity to study both of these as it can simultaneously measure both the $F$-region plasma distributions using GUVI and the tidal amplitudes at $E$-region altitudes using SABER (Forbes et al., 2006; Zhang et al., 2006). Here we shall investigate the correspondence of the tides retrieved from the SABER temperature observations with the longitudinal structure of the low-latitude airglow observed by GUVI. As the amplitude of different atmospheric tides at $E$-region altitudes vary considerably with season, it may be expected that the wavenumber-four pattern in the $F$-region should vary significantly with season. Using the tidal temperature amplitudes from SABER is a good proxy for the tidal zonal wind amplitudes that are believed to be responsible for the coupling between the tides at $E$-region altitudes and the $F$ region ionosphere (Hagan et al., 2007) as these parameters are coupled through geostrophic balance, so while there is a difference in their latitudinal structure, both vary in amplitude and zonal wavenumber in the same manner. Here we shall investigate the GUVI and SABER observations during vernal equinox as well as northern hemisphere summer and winter.

In the following section we shall use IMAGE FUV observations of the post-sunset airglow emissions to determine the changes in these emissions with LT. This is required prior to considering the TIMED GUVI data, which spans 20:00-02:00 LT. In Sections 3.1 and 3.2 we shall present our analysis of the TIMED GUVI airglow and the TIMED SABER temperature observations. In Section 4, we shall compare these two data sets for different seasons and demonstrate that essentially all of the large-scale longitudinal structure of the post-sunset $\mathrm{F}$-region $\mathrm{O}^{+}$distribution during magnetically quiet periods is created by the non-migrating tides at $E$-region altitudes.

\section{IMAGE FUV Observations}

The TIMED satellite is in a low-altitude, high-inclination orbit that precesses slowly in local time. In 30 days, the satellite moves through 6 hours of local time. Therefore, before we can use data from GUVI for a continuous 30 day period, we must first establish the local time evolution of the longitudinal morphology of the EIA. From the TIMED orbit, it is not possible to separate out local-time variations from variations associated with day-to-day changes in solar EUV flux and neutral winds. This makes GUVI unsuitable for accurately establishing the local-time evolution of the low-latitude ionosphere. This is illustrated in figure 2 of Henderson et al. (2005b), which shows a very complicated relationship between the magnetic latitude of the maximum airglow intensity and magnetic local time when the GUVI observations from 2002 and 2003 are used.

The high apogee orbit of the IMAGE satellite allowed the FUV instrument to observe the development of the morphology of the EIA across several hours of local time (up to 8 hours simultaneously) for several hours of universal time (up to 7 hours of continuous observations). Sagawa et al. (2005) used the IMAGE FUV data from 29 April-5 May 2002 to show the nighttime evolution of the morphology of the EIA (see their figure 4). They found that the four-peaked structure of the EIA in a constant local time frame is present throughout at least 20-02 LT, although some change in the location of the peaks over this LT range could be seen.

Here we shall investigate this relationship more thoroughly using an analysis of one month of IMAGE FUV data from 20 March-20 April 2002 (the same time interval considered in the previous studies highlighted in Section 1). We use the same data analysis as presented by England et al. (2006a). Briefly, the observed $135.6 \mathrm{~nm}$ airglow brightness in the northern airglow band is mapped into APEX magnetic coordinates (VanZandt et al., 1972) and then averaged over 15 minutes of universal time ( 8 images). The observed brightness is corrected for limb-brightening effects using a simple cosine relationship, which is reasonable for all included spacecraft zenith angles. Gaussian fits along magnetic meridians are made to data in bins of $1^{\circ}$ geomagnetic longitude. All data for conditions of $K_{\mathrm{p}} \geq 3$ are removed and then averaged in a $1^{\circ}$ geomagnetic longitude sliding window. The top panel of Fig. 1 shows the geomagnetic latitude of the peak airglow emissions as a function of geomagnetic longitude, offset by $72^{\circ}$, for one hour local time bins from 20-21 LT until 01-02 LT. This offset approximately co-locates the $0^{\circ}$ mark with $0^{\circ}$ geographic longitude, making the comparison to the tidal data presented in Section 3.2 and in previous works more straightforward. This coordinate system will be used for all of the ionospheric data presented in this work. The middle panel of Fig. 1 shows the peak brightness of the EIA. The bottom panel shows the number of estimates of the location and brightness that have been used in producing the first two panels. The data coverage for this period is generally good, apart from around $270^{\circ}$ longitude during the early evening. As discussed in England et al. (2006a), this is the longitude region where the spacecraft viewing geometry is the least ideal and there may still be some contribution from $135.6 \mathrm{~nm}$ dayglow in the observations close to the terminator. Thus, we shall restrict our discussion of the IMAGE FUV data to the peaks located around $30^{\circ}, 120^{\circ}$ and $210^{\circ}$ longitude. The most striking feature in Fig. 1 is the large change in the airglow brightness with local time shown in the middle panel. This is primarily a consequence of the total column amount of $\mathrm{O}^{+}$decreasing with local time due 

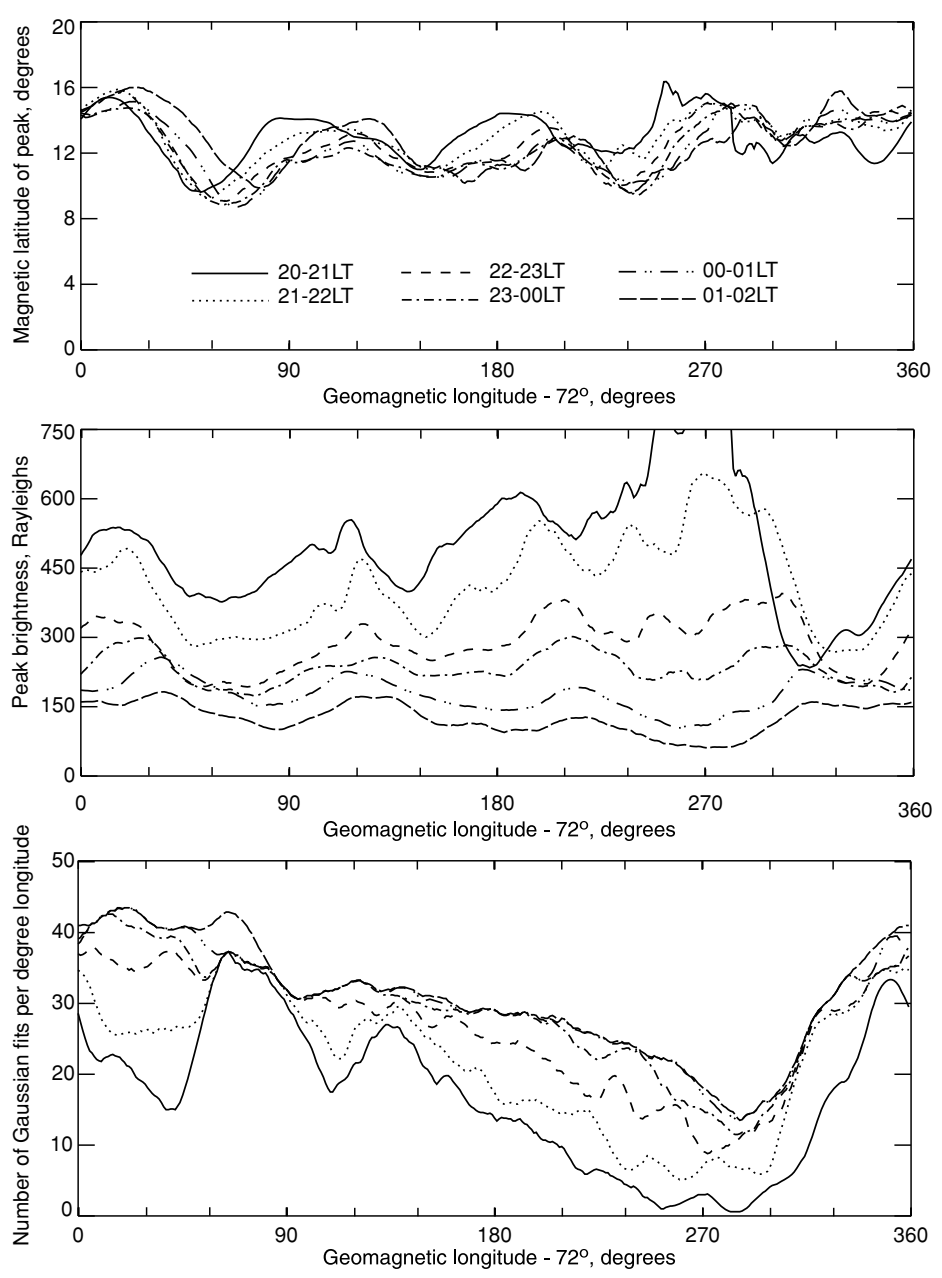

Fig. 1. (Top) The magnetic latitude of the peak airglow emissions of the northern $135.6 \mathrm{~nm}$ airglow arc as a function of geomagnetic longitude $-72^{\circ}$ for one hour local time bins from 20-21 local time until 01-02 local time. This shift approximately collocates the $0^{\circ}$ mark with $0^{\circ}$ geographic longitude to allow more easy comparison with other data sources and previous works. Data are from IMAGE FUV observations from 20 March-20 April 2002. Data have been processed using the method described by England et al. (2006a). (Middle) As top panel, but for the peak brightness of the airglow band. (Bottom) The number of estimates of the locations and brightnesses per degree longitude that have been used in producing the first two panels. The poor sampling of data around the $270^{\circ}$ longitude mark limits the usefulness of the IMAGE-FUV dataset in this region.

to charge exchange and recombination, although changes in electron temperature and $\mathrm{O}^{+}$scale height can also play a part (see Meier, 1991, for further details). The magnetic latitudes of the peak airglow brightness in the top panel show the wavenumber-four pattern to be present from 20-02 LT, with relatively small changes in these values throughout this local time interval. As these changes are small, and there is some uncertainty in the mapping of the IMAGE FUV data (see England et al., 2006a), we shall focus on the peak brightness data for a more detailed look at the changes in this wavenumber-four pattern with local time. Focusing on the peaks around $30^{\circ}, 120^{\circ}$ and $210^{\circ}$ longitude, a systematic shift of each peak to the east occurs with increasing local time. Each peak moves at $\sim 3-4^{\circ}$ longitude per hour. This matches the typical eastward plasma drift speed (Fejer et al., 1991) and the drift speed of depletions imbedded within the $\mathrm{O}^{+}$airglow observed by IMAGE FUV (Immel $e t$ al., 2003), for similar conditions. The purpose of this discussion is not to definitely establish that the eastward motion seen in the four-peaked structure is of the same origin as these previously observed drifts, but rather simply to note its existence and the need to account for this when combin- ing data that come from several hours of local time.

In the following section, we shall use 30 day intervals of GUVI $135.6 \mathrm{~nm}$ observations to study the morphology of the equatorial airglow bands. Intervals of this length are required to average out the strong day-to-day variations of the airglow bands and produce plots which are representative of a monthly mean and as such are suitable for comparison with the tidal temperature data from SABER. Over 30 days, the GUVI observations drift across 6 hours of local time (from 02 to $20 \mathrm{LT}$ ). In order to combine data from this wide range, we shall first remove the strong variation in absolute brightness with local time by normalizing to the same mean brightness. This shall produce usable mean brightness distributions representative of only the variations in the airglow as a function of latitude and longitude. The data shall also be shifted westward by $3^{\circ}$ longitude per hour of local time after 20:30 Local time. These corrections, discussed in more detail in the next section, allow easy comparison to previous works and comparison of observations from different time periods. It is worth noting here that a shift of around $3^{\circ}$ longitude per hour is small compared with the longitudinal structures in the ionosphere that are of order 
$90^{\circ}$ longitude or larger. While introducing this shift is justified as, there is no qualitative change in the results presented here by not omitting this step of the analysis.

\section{TIMED Observations}

\subsection{GUVI observations}

The TIMED GUVI instrument has been collecting nighttime airglow observations with nearly global coverage each day since early 2002. The most useful observations for studying the morphology of the equatorial airglow bands come from periods when the $135.6 \mathrm{~nm}$ airglow brightness is as high as possible. As the brightness of the $135.6 \mathrm{~nm}$ airglow emission varies as the line-of-sight integral of the square of the $\left(\mathrm{O}^{+}\right)$, the most suitable observations come from 2002-2003 when conditions were close to solar maximum. For these periods, the morphology of the equatorial airglow bands are clearly visible between sunset and 02 LT. After $02 \mathrm{LT}$, the airglow is too dim to be reliably detected in many cases. Prior to $20 \mathrm{LT}$, the uplift of the $F$-region ionosphere associated with the post-sunset rise is still taking place. As the time of this varies significantly with location (e.g. Scherliess et al., 1999), observations of the longitudinal morphology of the airglow bands in this local time period will be dominated by this variation. As this is not what we wish to study here, we shall confine our analysis to data from 20-02 LT.

Here we shall consider 30 day samples of the GUVI observations from three seasons. These are days 71-100 of 2002, which we shall refer to as March hereafter; days 191-220 of 2002, which we shall refer to as July hereafter and days 8-37, of 2003 which we shall refer to as January hereafter (January 2002 contains insufficient data for our analysis). In order to study the morphology of the lowlatitude airglow bands in the absence of external penetrating electric fields, data in each of these seasons are sorted for low to moderate geomagnetic activity $\left(K_{\mathrm{p}} \leq 4\right)$. This is slightly less strict than the sorting of the IMAGE FUV data in the previous section, or the previous TIMED GUVI analyses discussed in the introduction which sorted for $K_{\mathrm{p}}$ $\leq 3$, but is required to allow a sufficient number of observations in each of these time periods to produce plots representative of the monthly mean airglow morphologies. Additionally, unlike the previous TIMED GUVI analyses, we shall not follow the analysis technique of Henderson et al. (2005a), which was restricted to only nadir data (a small fraction of the disc data collected by GUVI). Henderson et al. (2005a)'s analysis technique was able to remove the effects of plasma bubbles on the observed airglow brightness and find the peak brightness and magnetic latitude of that peak every $15^{\circ}$ magnetic longitude. As we wish to consider longer data intervals than used in the previous GUVI analyses, the effects of plasma bubbles are less significant and as such we shall use GUVI observations from all sub-limb angles that are not contaminated with thermospheric dayglow. This shall increase the total number of observations upon which our results shall be based, but introduces some inaccuracies in our analysis from the uncertainty in the altitude from which individual emissions originate. Therefore, we do not expect the details of our analysis to agree with those presented by Henderson et al. (2005b) and will not focus
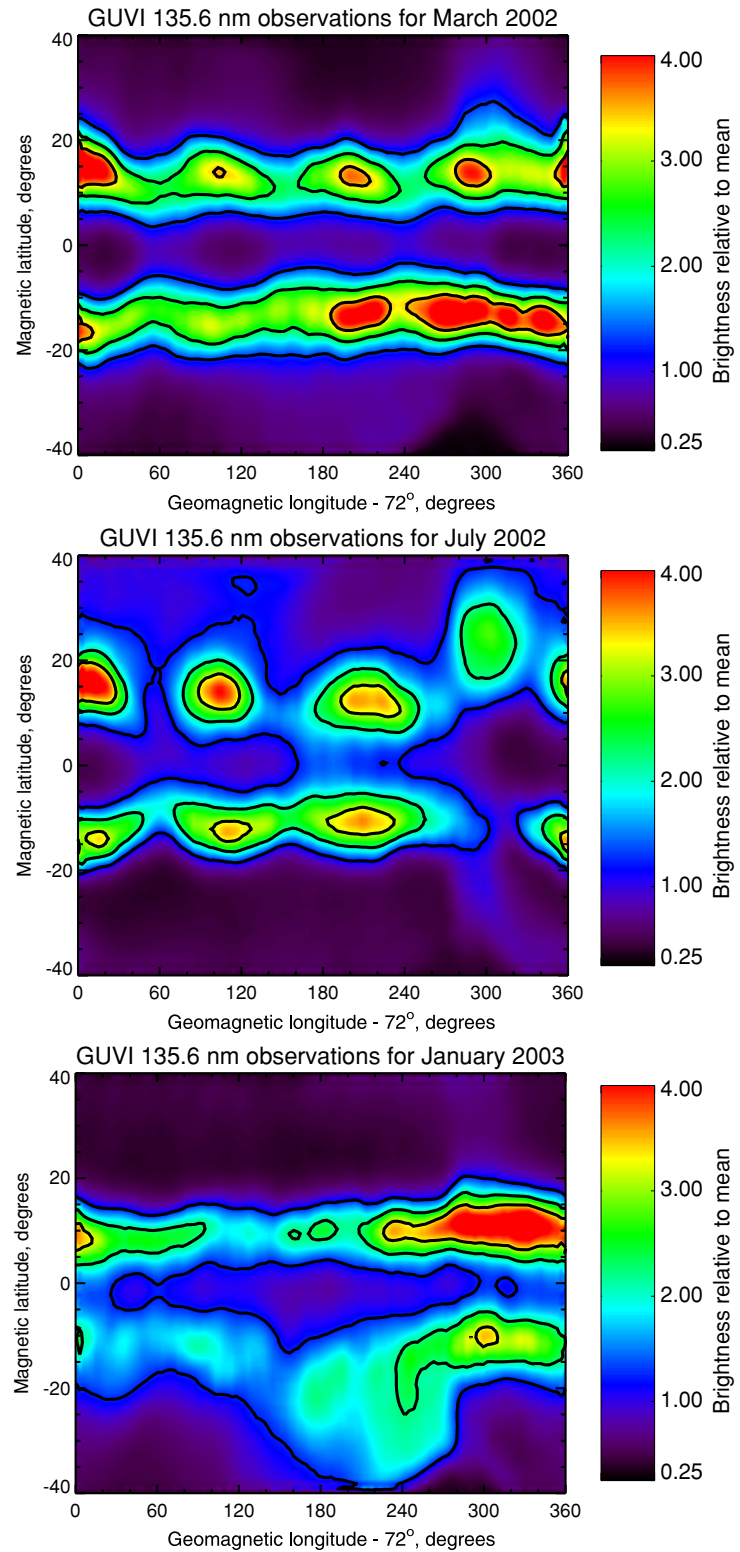

Fig. 2. GUVI $135.6 \mathrm{~nm}$ observations for (top) March, (middle) July and (bottom) January for magnetically quiet periods $\left(K_{\mathrm{p}} \leq 4\right)$ in geomagnetic coordinates, offset by $72^{\circ}$. This shift approximately collocates the $0^{\circ}$ mark with $0^{\circ}$ geographic longitude to allow more easy comparison with other data sources and previous works. Positions represent the morphology of the airglow bands at 20:30 LT. Contours are shown at values of 1, 2 and 3 times the mean value (unity). See text for details of the analysis and data used.

on such differences in this work. However, as the viewing geometry of GUVI is identical for each $15^{\circ}$ longitude orbit scan and as $15^{\circ}$ is small compared with the longitudinal structure we wish to study $\left(\sim 90^{\circ}\right)$, such inaccuracies will not significantly impact our results.

For our analysis we first take all of the GUVI observations for one season with $K_{\mathrm{p}} \leq 4$. These observations are mapped APEX magnetic coordinates. This produces a single global map per day of observations. We shall then follow the analysis steps discussed in Section 2. The values of the emissions in each of these daily maps are normalized by dividing by the mean brightness. These are then shifted westward by $3^{\circ}$ magnetic longitude per hour local time after 

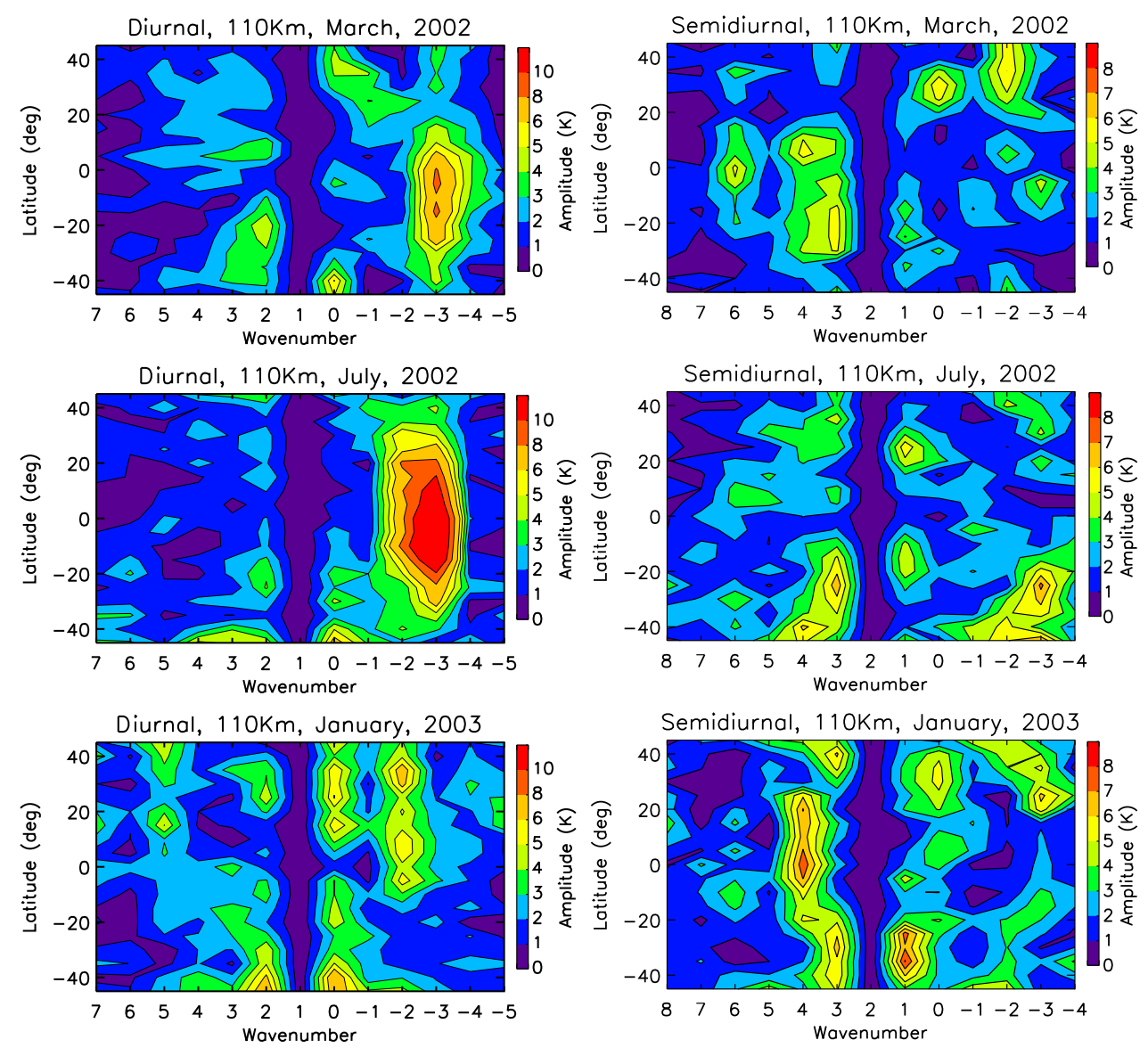

Fig. 3. SABER tidal spectra for non-migrating tides versus latitude at $110 \mathrm{~km}$ for (top) March 2002, (middle) July 2002 and (bottom) January 2003. Values shown are diurnal amplitudes in Kelvin. Positive wavenumbers correspond to westward propagating waves and negative wavenumbers correspond to eastward propagating waves. The migrating modes-westward wavenumber 1 diurnal and wavenumber 2 semidiurnal have been suppressed.

20:30 LT and then summed and binned into a single array with a resolution of $3^{\circ}$ magnetic longitude by $2^{\circ}$ magnetic latitude. The array is then divided by the number of days included in this analysis. This final array of corrected mean brightnesses represents the latitudinal and longitudinal variations in the airglow brightness, has a mean value of 1 regardless of the solar EUV emissions and the data from each day included within it carry equal weight.

Figure 2 shows the processed GUVI data for March, July and January in magnetic latitude-longitude coordinates, offset by $72^{\circ}$ magnetic longitude (see Section 2 ). Contours are shown at values of 1,2 and 3 times the mean value.

\subsection{TIMED SABER observations}

The TIMED SABER instrument measures kinetic temperatures from $\mathrm{CO}_{2}$ emissions from $20-120 \mathrm{~km}$ altitude. Here we use the same tidal analysis as Forbes et al. (2006), which separates the tidal temperature fluctuations into the contributions from different tidal components (both diurnal and semidiurnal for wavenumbers from 6 west to 6 east) for 60 day intervals of data centered on the 15 day of each month. As the SABER observations provide complete longitudinal coverage and latitudinal coverage up to $50^{\circ}$, this is ideal for studying the important non-migrating tidal components in the lower thermosphere which are believed to influence the longitudinal structure of the EIA as measured by GUVI. The SABER data used here are Version 6 , as opposed to Version 4 used by Forbes et al. (2006), but in all other respects our analyses are identical. Here we shall use two products of the analysis described by Forbes et al. (2006). The first are spectra for the non-migrating diurnal and semi-diurnal tides at $110 \mathrm{~km}$. These show the amplitude of each wave component as functions of latitude and wavenumber (see figure 2 of Forbes et al. (2006) and related discussion). Tidal fluctuations at altitudes around $110 \mathrm{~km}$ are believed to have the greatest impact on the EIA because this region is close to the peak in $E$-region Hall and Pedersen conductivities (110 and $130 \mathrm{~km}$ respectively) and is also close to the altitude where the amplitude of the DE3 tide is believed to maximize. Figure 3 shows these spectra for March, July and January.

We shall also present the residuals from the mean temperatures measured by SABER as a function of latitude and longitude at $110 \mathrm{~km}$ altitude (see figure 6 of Forbes et al. (2006) and related discussion). These are shown in Fig. 4 for the same intervals as the GUVI data presented in Section 3.1. These show the averages of the same 30 days period of observations as the GUVI data and are for the ascending portion of the TIMED orbit only. During this time, the observations sweeps through six hours of local time ( 02:30-20:30 LT) and as such there is some cancellation of the amplitude of persistent wave-features and as such we shall not focus on the actual temperature values reported 

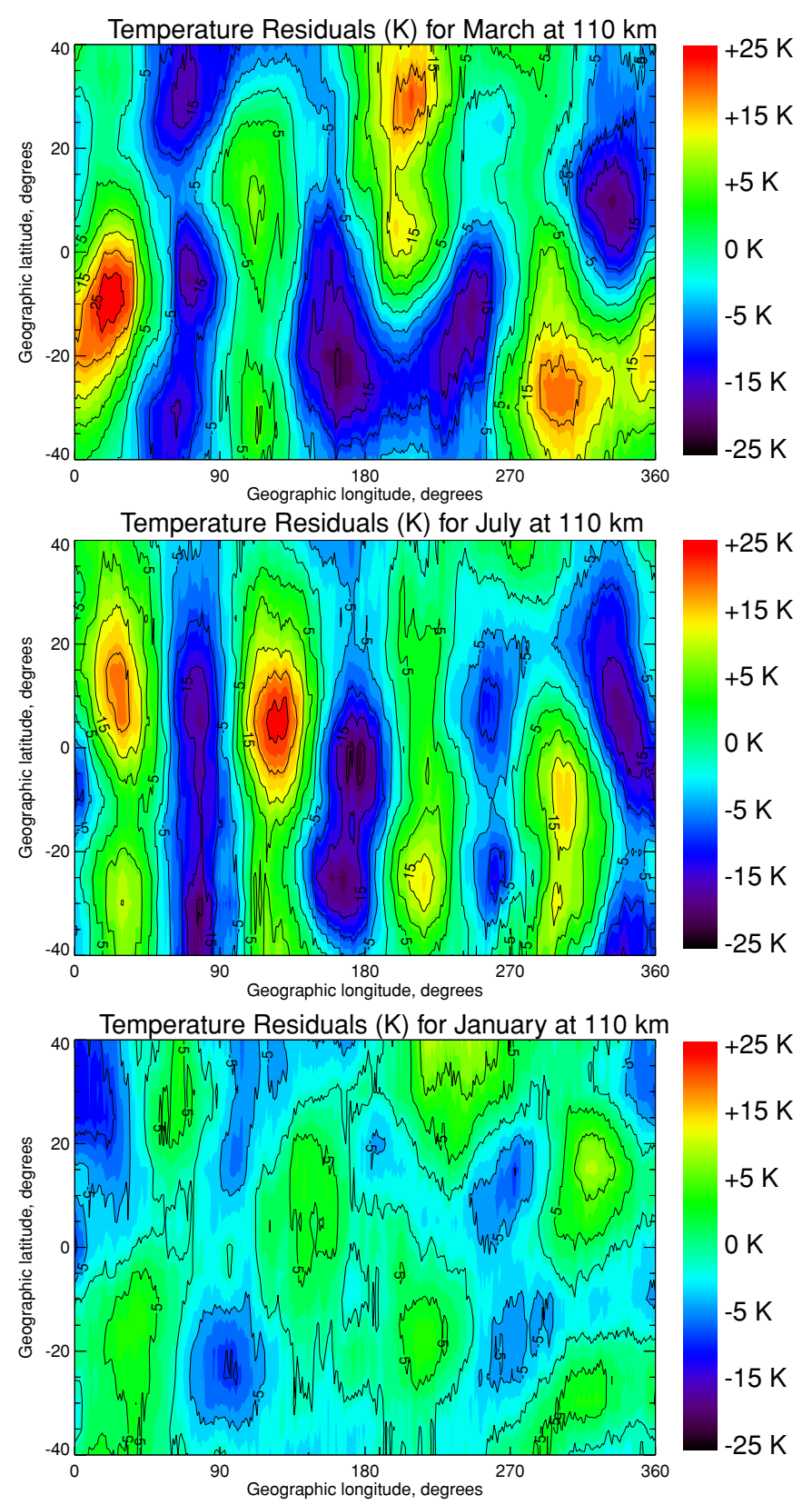

Fig. 4. 30 day mean residuals from mean temperatures as a function of longitude and latitude measured by SABER at $110 \mathrm{~km}$ altitude for the same date ranges as used for the GUVI data in Figs. 2 and 3. As in Fig. 3, the migrating modes have been suppressed. Data come from 20:30-02:30 local time.

in this figure. Nevertheless, these are instructive as they both confirm what feathers are present in the temperatures at $110 \mathrm{~km}$ altitude during the 30 day period considered in the GUVI observations and they are useful for establishing the relative position (latitude and longitude) of such features for the three time periods considered. Figure 5 shows the wavenumber-spectra of these data, that show what strong wavenumber features exist. For just 30 days of SABER data, it is not possible to separate these out into east and west components, so we shall rely on Fig. 3 for this information.

\section{Discussion}

\subsection{Comparison of March with July}

Figure 6 of Zhang et al. (2006) shows that the amplitude of the DE3 tide varies strongly with season. As this tidal component is believed to be the main driver in creating the wavenumber-four patterns observed in the equatorial $F$-region ionosphere, we may expect the amplitude of this wavenumber-four pattern to vary in the same way. The amplitude of the DE3 maximizes around July-September, minimizes around January-February and has an intermediate value around March-April.

We shall begin by considering the GUVI observations for March and July shown in Fig. 2. A wavenumber-four signature is visible in both the northern and southern airglow arcs for both seasons, although it is stronger in the northern arc in both cases. Indeed during March, the two peaks in the southern arcs expected around $270^{\circ}$ and $360^{\circ}$ longitude appear as a single extended peak and in July the peak expected around $270^{\circ}$ is a much lower amplitude than those at other locations. The wavenumber-four pattern is substan- 

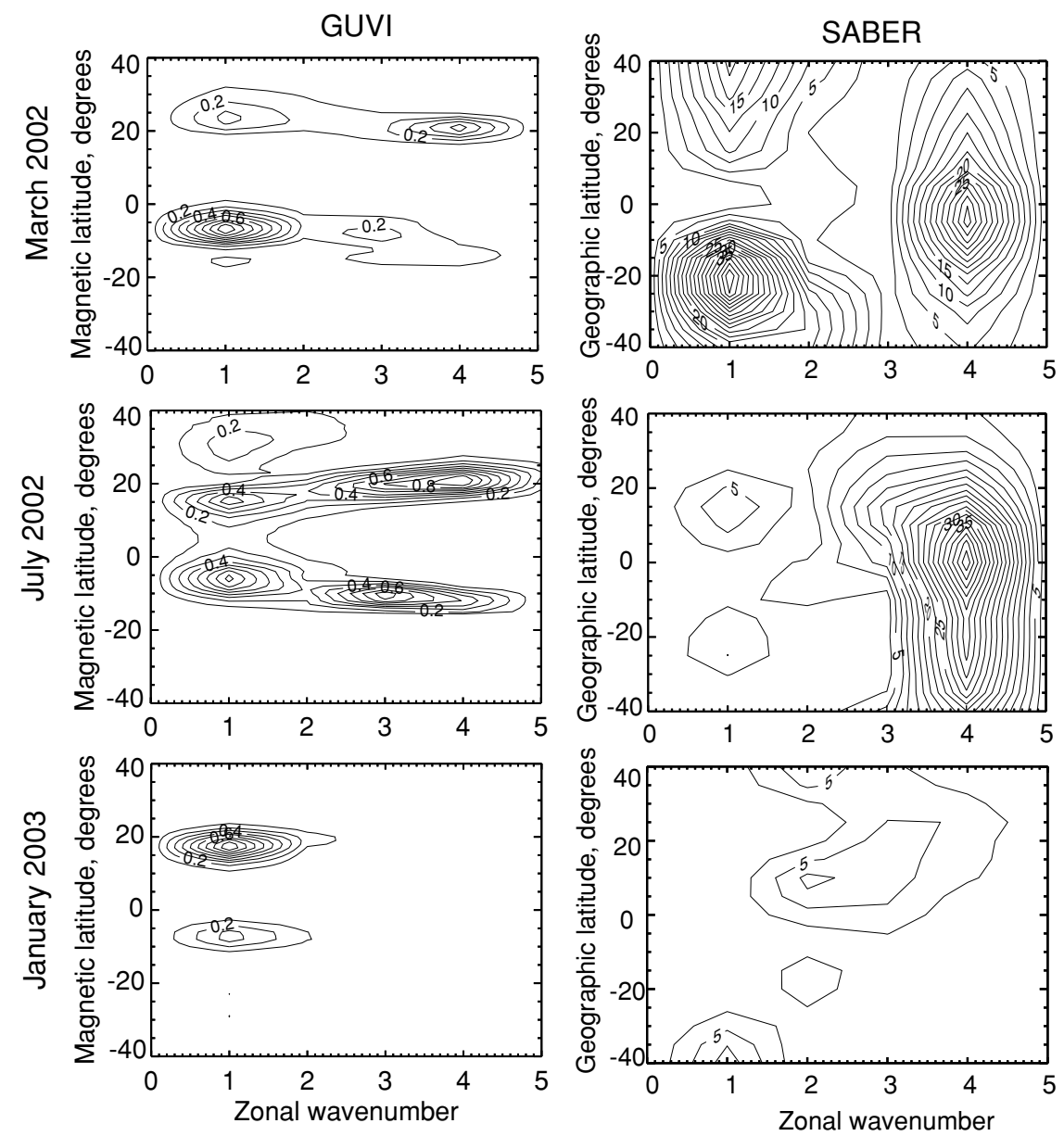

Fig. 5. Zonal wavenumber spectra for (left) the airglow brightness observed by GUVI shown in Fig. 2 and (right) the temperature residuals from SABER shown in Fig. 4. Spectra are shown as a function of (left) magnetic latitude and (right) geographic latitude for (top) March 2002, (middle) July 2002 and (bottom) January 2003. These spectra show the power in each wavenumber component only, and do not separate these out into their contributions from individual eastward and westward propagating waves. For both, the wavenumber-zero component (zonal mean and the migrating modes respectively) has been suppressed. Units for GUVI are relative to the peak in the spectrum with a contour interval of 0.2 . Units for the SABER data are $\mathrm{K}^{2}$ with a contour interval of $2.5 \mathrm{~K}^{2}$.

tially stronger in the July observations than the March observations. As the monthly mean $F_{10.7}$ fluxes are almost the same for these months, we can eliminate this as the cause of the observed difference. As shown in Fig. 3, the amplitude of the DE3 tide is substantially greater around July than March. Therefore the increase in the amplitude of the wavenumber-four pattern observed by GUVI is consistent with a change in the non-migrating component believed to be responsible for this pattern.

For a more quantitative look at the longitudinal structures observed by GUVI during March and July, we plot the wavenumber spectra for these airglow emissions in Fig. 5 (left). During March, the strongest features in this spectrum are wavenumbers one and four. The spectrum of the diurnal tides (Fig. 3, left) shows that the DE3 is the strongest tidal component present at $E$-region altitudes and this has already been linked with the wavenumber-four features in the airglow seen during equinox (see Section 1). This is also seen in the temperature residuals wavenumber spectrum (Fig. 5, right). The other tides that appear as wavenumberfour structures in fixed local time (the diurnal westward five DW5, semi-diurnal westward six SW6 and semi-diurnal eastward two SE2) all have low amplitude during this time period, so can be ruled out as the main cause of this feature in the airglow. The strong wavenumber-one feature seen in the GUVI data is not readily explained by any of the diurnal tidal components. While both the DW2 and D0 (diurnal standing wave) appear as wavenumber-one features in fixed local time, both of these were low amplitude during this period. However, the temperature residual wavenumberspectrum shows that a strong wavenumber-one is present at $E$-region altitudes. This feature is therefore likely associated with the forcing of a non-migrating semi-diurnal tide. Both the SW1 and SW3 appear as wavenumber-one features in fixed local time. The primary mode of the SW3 is symmetrical and is seen here to have a large amplitude in Fig. 3, especially in the southern-hemisphere. This is therefore a likely candidate for producing the wavenumber-one features observed in the $F$-region $\mathrm{O}^{+}$layer during March. It is possible that the variation in the magnetic field with longitude also plays a role in creating the wavenumber-one feature, but it is not clear why this would produce the observed hemispheric asymmetry in the airglow signature-a feature that does mirror the power distribution of the SW3.

The strong asymmetry of both the wavenumber-one and wavenumber-four signatures is unexpected and is not read- 

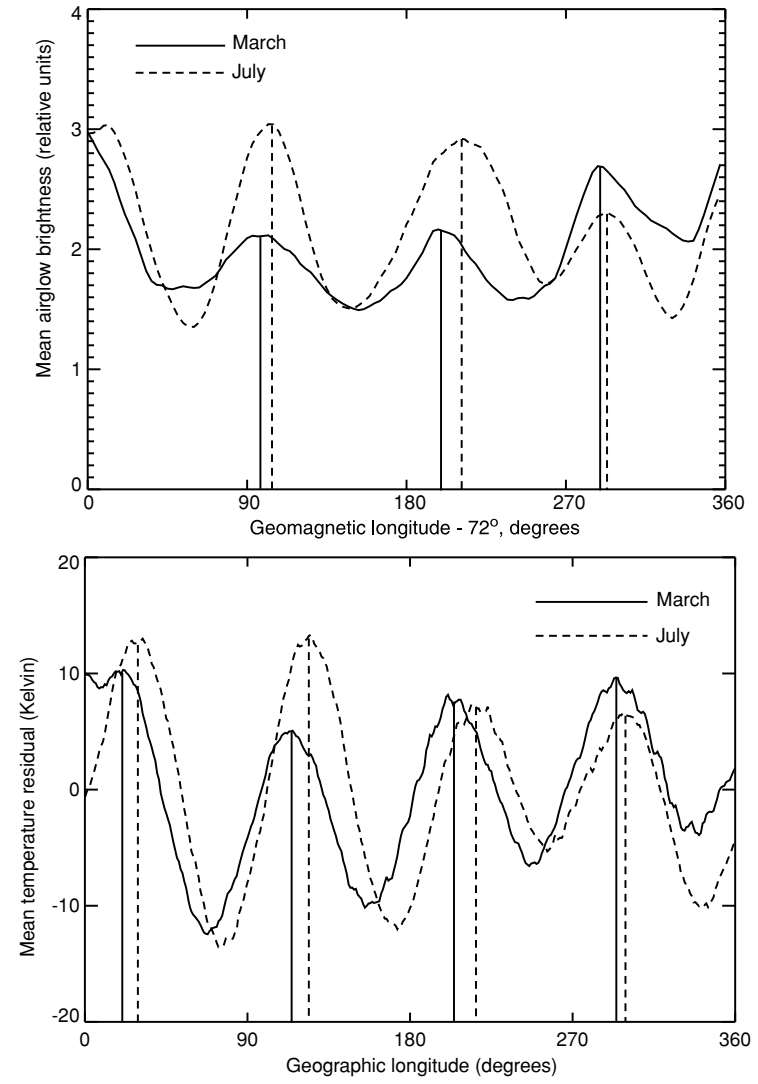

Fig. 6. (Top) Mean airglow brightness in the northern arc averaged over 0-20 magnetic latitude and (bottom) mean temperature residual from the SABER data shown in Fig. 4 averaged over $-40-40^{\circ}$ geographic latitude. The solid line represents March data and the dashed line represents July data. Values are in the same relative units as used in Fig. 2. The airglow data have been smoothed over $21^{\circ}$ geomagnetic longitude and the temperature residuals have been smoothed over $21^{\circ}$ geographic longitude. The airglow data have been shifted by $72^{\circ}$ geomagnetic longitude as in previous figures. The vertical lines are for visual guidance.

ily explained by other factors such as inter-hemispheric winds as these are generally small around equinox (as is evident by the lack of asymmetry in the total airglow brightness). The GUVI wavenumber spectrum shown in Fig. 5, that analyzes only the brightness of the airglow as a function of latitude, does not show a wavenumber-four signature in the southern hemisphere. However, a wavenumber-four signature is visible in the location of the southern airglow arc shown in Fig. 2. This especially clear in the $0-240^{\circ}$ longitude region.

During July, the wavenumber-four signature is the strongest feature in both the GUVI and SABER wavenumber-spectra shown in Fig. 5. This is consistent with the strong DE3 observed by SABER during this time period (Fig. 3). Again, no other tidal component exists at a significant amplitude that could create this feature. The second most prominent feature in the GUVI wavenumber spectrum is a wavenumber-three signature in both hemispheres. The DE2 is known to have a phase structure and vertical wavelength that would make it efficient at driving $F$-region longitudinal variations through the same process as described for the DE3 (e.g. Forbes et al., 2008). The DE2 would produce a wavenumber-three structure in a con- stant local time frame. Figure 3 shows that the amplitude of this wave is also high during July (almost as high as the DE3) and as such it seems likely that this tide may account for the observed wavenumber-three signature. Further, the lack of a wavenumber-three signature in March, when the DE2 is essentially absent also points to this wave, rather than some permanent, static feature as being responsible for the signature seen in the $F$-region ionosphere. It is not clear why the wavenumber-three signature does not appear strongly in the southern hemisphere for the temperature residuals wavenumber spectrum in Fig. 5, although some asymmetry in the DE2 power between the two hemispheres is also seen in Fig. 3. Other candidate tides that appear as wavenumber-three in fixed local time (DW4, SW5 and SE1) are all low amplitude during July.

A significant wavenumber-one signature is seen in the GUVI data during July that is similar, but slightly lower in amplitude than during March. Unlike during March, this does not appear strongly in the temperature residuals wavenumber spectrum in Fig. 5 (right). From Fig. 3, there is some power in SW3, although its power is seen to minimize at the equator, which is a signature of the 1st antisymmetric mode, rather than the symmetric mode discussed earlier. A consequence of the high field-aligned conductivity in the ionosphere is that asymmetric wind patterns are not effective at modifying the dynamo fields (e.g. Richmond and Roble, 1987) and as such this is unlikely to be the source of the wavenumber-one observed in the $F$-region airglow emissions. Alternatively, a wavenumber-one signature may be expected in the observed FUV brightness around solstice as a result of the large variation in the distance between the sub-solar point and magnetic equator. Wavenumberone signatures may also arise from the offset of the magnetic and geographic equators, the declination of magnetic field lines in a geographic frame of reference and variations in the strength of the magnetic field with longitude (e.g. Walker, 1981).

A closer examination of the locations of the peaks in the GUVI observations can be achieved by plotting the mean brightness of the equatorial airglow, averaged over 0-20 magnetic latitude (where the pattern is strongest) and smoothed over $21^{\circ}$ longitude to remove small-scale features and noise. This is shown in Fig. 6 (top) for March and July. This shows that the wavenumber-four pattern is shifted eastward in July compared to March, with a mean shift of $8^{\circ}$ longitude. An equivalent plot can be made for the SABER temperature residuals. Figure 6 (bottom) shows the mean temperature residuals as a function of longitude, averaged over $-40-40^{\circ}$ geographic latitude and again smoothed over $21^{\circ}$ longitude. The wavenumber-four pattern in the temperature residuals (related to the DE3), is also shifted eastward by $9^{\circ}$ in July compared to March as a result of a change in the phase of this wave at $110 \mathrm{~km}$ altitude between these two months. This shift is created by differences in propagation of the DE3 as it travels from its source in the lower atmosphere up to $110 \mathrm{~km}$. As the data for the SABER temperatures come from 20:30-02:30 LT, one would not expect an exact correspondence in the locations of the four peaks in the temperatures and airglow as one represents an instantaneous forcing on the $E$-region and the other represents 
the time-integrated effects of this forcing (see England et al., 2006a). Indeed, performing a check of the correspondence of the longitudes of the peaks in the tides and the airglow requires a consideration of the time-integrated effects of these tides and ion production, loss and transport that is better left to coupled modeling studies such as Hagan et al. (2007). However, it is reasonable to assume that any change phase of the tides between March and July should be consistent with a change in the positions of the airglow peaks observed during those two periods. Here, the mean shift is $9^{\circ}$ longitude, which is very close to the $8^{\circ}$ shift seen in the GUVI data, strongly implying that the DE3 is responsible for creating the longitudinal structure of the EIA and associated airglow bands.

Further examination of Fig. 2 reveals that the airglow arcs are shifted northwards in July compared with March (especially in the northern hemisphere). This can be understood in terms of the change in the sub-solar point and $F$-region meridional winds (that alter the $F$-region altitude) between near equinox and near solstice conditions and is similar to the changes that have been reported in the total electron content of the EIA region (TEC; Jee et al., 2004). As the $135.6 \mathrm{~nm}$ nightglow depends strongly on the total column amount of $\mathrm{O}^{+}$, the airglow arcs respond to this seasonal change in the same manner as TEC. It is worth noting here that this is opposite to the behavior of the $630.0 \mathrm{~nm}$ redline emission which is also associated with $\mathrm{O}^{+}$. This emission is strongly dependent upon the altitude of the $F$-region peak (decreasing in brightness with increasing altitude) and as such shows the opposite seasonal variation (Thuillier et al., 1976, 2002). The impact of this latitudinal shift on the $135.6 \mathrm{~nm}$ nightglow may also be the cause for the apparent absence of the peak around $300^{\circ}$ longitude. The magnetic equator lies around $15^{\circ}$ south of the geographic equator in this region and so the southern airglow band is furthest from the sub-solar point here, and thus we may expect low emission rates here. It is possible that the absence of this peak in only one hemisphere may account for the hemispheric asymmetry in the power of the wavenumber-four signature shown in Fig. 5 during this period.

\subsection{Comparison of March and July with January}

Figure 6 of Zhang et al. (2006) shows that the amplitude of the DE3 tide has a minimum around January-February. This is therefore an ideal time to look at the morphology of the airglow arcs when the DE3 may not be expected to dominate their longitudinal structure. The GUVI observational period that most closely corresponds to this time period is days 8-37 for 2003 (there was insufficient data gathered in 2002). For this time period, the solar EUV flux was lower than for the 2002 periods, but was still moderately high (the mean $F_{10.7}$ flux was $140 \times 10^{-22} \mathrm{~J} \mathrm{~s}^{-1} \mathrm{~m}^{-2} \mathrm{~Hz}^{-1}$ for January compared with $180 \times 10^{-22} \mathrm{~J} \mathrm{~s}^{-1} \mathrm{~m}^{-2} \mathrm{~Hz}^{-1}$ in both March and July).

Figure 2 shows that the wavenumber-four pattern is almost completely gone in January (although some evidence of a weak wavenumber-four pattern can still be seen in the southern airglow arc with peaks in approximately the same locations as those in March and July). This is as expected from the amplitude of the DE3 is very low at this time ( $2 \mathrm{~K}$ compared with over $10 \mathrm{~K}$ in July).
The airglow wavenumber spectrum shown in Fig. 5 highlights the dominance of the wavenumber-one component in the GUVI observations. This is clearly visible in Fig. 2, with the bright region (high $\mathrm{O}^{+}$density) extending from $240^{\circ}-0^{\circ}$ longitude in both hemispheres, although it is slightly more pronounced in the north. This compares favorably with the TEC observations from TOPEX reported by Scherliess et al. (2008) for the 21-24 local time period for November-February. However, care must be taken in comparing these two sets of observations as the TOPEX data were for an average of 4 months, compared with the 1 month used here, and it is evident from analyses of the tidal data from SABER cited above that the dominant non-migrating tidal components can vary significantly over a 4 months period. A wavenumber-one component is not seen in the temperature residuals wavenumber spectrum in Fig. 5 and as such it seems unlikely that the wavenumber-one seen in the airglow is created by the $E$ region non-migrating tides. Examining these more closely, the DW2, D0 and SW3 are all low amplitude and SW1 only achieves significant amplitude in the southern hemisphere, polewards of $30^{\circ}$. Hagan and Forbes (2003) have shown that this is associated with a mode that maximizes at the poles. Additionally, the impact of the asymmetry in the power distribution of this wave on its ability to modify the ionosphere is not well understood. Further study of the effects of strong asymmetry in the wavenumber power distributions of atmospheric tides on the ionosphere using coupled thermosphere-ionosphere models is required. It seems likely that the strong wavenumber-one signature seen in the airglow wavenumber spectrum is related to geometric and magnetic field effects during this period, as described for the July case, and not to the non-migrating tides in the $E$ region.

As with July, the DE2 is seen in the SABER tidal spectrum for January. During July, this wave was seen to be effective in creating a wavenumber-three pattern in the airglow, but such a pattern is absent in the January data. During January, the power of this tide is entirely concentrated within the northern hemisphere, which may explain the absence of this feature in the airglow data.

Unlike March and July, SW4 reaches a significant amplitude during January, peaking at $8 \mathrm{~K}$ at the equator. This power in this component is slightly asymmetrical with a higher power in the north than the south, although significant amplitudes are observed in both hemispheres. This wave has been observed to have a vertical wavelength $\sim 37 \mathrm{~km}$ and Forbes et al. (2008) have argued that it should be relatively efficient in creating a wavenumber-two structure in the ionosphere. There is almost no power in the wavenumber-two component of the GUVI wavenumber spectrum in Fig. 5, which is consistent with the lack of a wavenubmer-two in the temperature residuals for this period. It seems likely that this wave is only significant during another portion of the 60-day window included in Fig. 3. Further study, perhaps using other time periods may reveal more about the potential influence of this tidal component on the morphology of the ionosphere, but that is beyond the scope of this study.

The magnetic latitude of both airglow arcs is seen to 
move south in both hemispheres, with the southern arc visible to almost $-40^{\circ}$ magnetic latitude around $200^{\circ}$ longitude. This is again consistent with the changes in subsolar point and $F$-region meridional winds by which we explained the July observations.

\section{Conclusions}

We have shown that:

1) The wavenumber-four pattern observed in the equatorial $F$-region ionosphere by GUVI during March is consistent with the driving tidal component (DE3) observed by SABER at $E$-region altitudes during the same time period.

2) Changes in the amplitude of the DE3 at $E$-region altitudes with season (both increases and decreases) are reflected in changes in the amplitude of the wavenumber-four pattern in the $F$-region ionosphere.

3) The shift in longitude of the location of the peaks in the temperature residuals observed by SABER between March and July is also seen in the GUVI observations of the ionosphere. This again demonstrates that the DE3 is responsible for producing the wavenumberfour pattern observed in the $F$-region ionosphere.

4) During March, a wavenumber-one signature is seen in the airglow. This may be explained by some combination of the SW3 tide observed at $E$-region altitudes and changes in the magnetic field with longitude.

5) During July, there is evidence of a significant wavenumber-three pattern in the airglow observations that has not previously been reported. This is consistent with driving by the DE2 tide which attains a significant amplitude during this period.

6) Around both solstices, a strong wavenumber-one signature is also seen in the ionosphere which is not seen in the temperature residuals at $E$-region altitudes. It seems likely that this arises instead from changes in the magnetic field and sub-solar point as a function of longitude, both of which have wavenumber-one components.

7) The absence of any wavenumber-three pattern in the airglow during January may be because the amplitude of the DE2 is concentrated in the northern hemisphere. The impact of hemispheric asymmetry in both the forcing by non-migrating tides and in the longitudinal structure in the airglow they create requires further study.

8) Latitudinal shifts in the locations of the airglow arcs observed by GUVI follow the same pattern as those in TEC and both can be explained by changes in the sub-solar point and $F$-region meridional winds.

Acknowledgments. The IMAGE FUV analysis is supported by NASA through Southwest Research Institute subcontract number 8382 at the University of California Berkeley under contract NAS5-96020. The TIMED GUVI analysis is supported by the NASA Guest Investigator program. J. Forbes and X. Zhang acknowledge support under grants NNX07AB74G and NAG5-5028 from the NASA TIMED Program to the University of Colorado.

\section{References}

Appleton, E., Two anomalies in the ionosphere, Nature, 157, 691, 1946. England, S. L., T. J. Immel, E. Sagawa, S. B. Henderson, M. E. Hagan, S. B. Mende, H. U. Frey, C. M. Swenson, and L. J. Paxton, Effect of atmospheric tides on the morphology of the quiet time, postsunset equatorial ionospheric anomaly, J. Geophys. Res., 111, A10S19, doi:10.1029/2006JA011795, 2006a.

England, S. L., S. Maus, T. J. Immel, and S. B. Mende, Longitudinal variation of the $E$-region electric fields caused by atmospheric tides, Geophys. Res. Lett., 33, doi:10.1029/2006GL027465, 2006 b.

England, S. L., T. J. Immel, and J. D. Huba, Modeling the longitudinal variation in the post-sunst far-ultraviolet OI airglow using the SAMI2 model, J. Geophys. Res., 113, A01309, doi:10.1029/2007JA012536, 2008

Farley, D. T., E. Bonelli, B. G. Fejer, and M. F. Larsen, The prereversal enhancement of the zonal electric field in the equatorial ionosphere, $J$. Geophys. Res., 91, 13,723-13,728, 1986.

Fejer, B. G., E. R. de Paula, S. A. Gonzalez, and R. F. Woodman, Average vertical and zonal $F$ region plasma drifts over Jicamarca, J. Geophys. Res., 96, 13,901-13,906, 1991.

Forbes, J. M., J. Russell, S. Miyahara, X. Zhang, S. Palo, M. Mlynczak, C. J. Mertens, and M. E. Hagan, Troposphere-thermosphere tidal coupling as measured by the SABER instrument on TIMED during July-September 2002, J. Geophys. Res., 111, A10S06, doi:10. 1029/2005JA011492, 2006.

Forbes, J. M., X. Zhang, S. Palo, J. Russell, C. J. Mertens, and M. Mlynczak, Tidal variability in the ionospheric dynamo region, J. Geophys. Res., 113, A02310, doi:10.1029/2007JA012737, 2008.

Hagan, M. E. and J. M. Forbes, Migrating and nomigrating semidiurnal tides in the upper atmosphere excited by tropospheric latent heat release, J. Geophys. Res., 108(A2), 1062, doi:10.1029/2002JA009466, 2003.

Hagan, M., A. Maute, R. G. Roble, A. D. Richmond, T. J. Immel, and S. L. England, Connections between deep tropical clouds and the Earth's ionosphere, Geophys. Res. Lett., 34, L20109, doi:10.1029/2007 GL030142, 2007.

Hartman, W. A. and R. A. Heelis, Longitudinal variations in the equatorial vertical drift in the topside ionosphere, J. Geophys. Res., 112, A03305, doi:10.1029/2006JA011773, 2007.

Henderson, S. B., C. M. Swenson, J. H. Gunther, A. B. Christensen, and L. J. Paxton, Method for characterization of the equatorial anomaly using image subspace analysis of Global Ultraviolet Imager data, J. Geophys. Res., 110, A08308, doi:10.1029/2004JA010830, 2005a.

Henderson, S. B., C. M. Swenson, A. B. Christensen, and L. J. Paxton, Morphology of the equatorial anomaly and equatorial plasma bubbles using image subspace analysis of Global Ultraviolet Imager data, $J$. Geophys. Res., 110, A11306, doi:10.1029/2005JA011080, 2005b.

Immel, T. J., S. B. Mende, H. U. Frey, L. M. Peticolas, and E. Sagawa, Determination of low latitude plasma drift speeds from FUV images, Geophys. Res. Lett., 30(18), 1945, doi:10.1029/2003GL017573, 2003.

Immel, T. J., E. Sagawa, S. L. England, S. B. Henderson, M. E. Hagan, S. B. Mende, H. U. Frey, C. M. Swenson, and L. J. Paxton, Control of equatorial ionospheric morphology by atmospheric tides, Geophys. Res. Lett., 33, L15108, doi:10.1029/2006GL026161, 2006.

Jee, G., R. W. Schunk, and L. Scherliess, Analysis of TEC data from the TOPEX/Poseidon mission, J. Geophys. Res., 109, A01301, doi:10. 1029/2003JA010058, 2004.

Kil, H., S. J. Oh, M. C. Kelley, L. J. Paxton, S. L. England, E. Talaat, K. W. Min, and S. Y. Su, Longitudinal structure of the vertical $\mathbf{E} \times \mathbf{B}$ drift and ion density seen from ROCSAT-1, Geophys. Res. Lett., 34, L14110, doi:10.1029/2007GL030018, 2007.

Lin, C. H., W. Wang, M. E. Hagan, C. C. Hsiao, T. J. Immel, M. L. Hsu, J. Y. Liu, L. J. Paxton, T. W. Fang, and C. H. Liu, Plausible effect of atmospheric tides on the equatorial ionosphere observed by the FORMOSAT3/COSMIC: Three-dimensional electron density structures, Geophys. Res. Lett., 34, L11112, doi:10.1029/2007GL029265, 2007.

Meier, R. R., Ultraviolet spectroscopy and remote sensing of the upper atmosphere, Space Sci. Rev., 58, 1-185, 1991.

Namba, S. and K. I. Maeda, Radio Wave Propagation, p. 86, Corona Publishing, Tokyo, 1939.

Richmond, A. D. and R. G. Roble, Electrodynamic effects of thermospheric winds from the NCAR thermospheric general circulation model, J. Geophys. Res., 92, 12365-12376, 1987.

Rishbeth, H., The F-layer dynamo, Planet. Space Sci., 19, 263-267, doi:10.1016/0032-0633(71)90205-4, 1971.

Sagawa, E., T. J. Immel, H. U. Frey, and S. B. Mende, Longitudinal structure of the equatorial anomaly in the nighttime ionosphere 
observed by IMAGE/FUV, J. Geophys. Res., 110, A11302, doi:10. 1029/2004JA010848, 2005.

Scherliess, L. and B. G. Fejer, Radar and satellite global equatorial $F$ region vertical drift model, J. Geophys. Res., 104, 6829-6842, doi:10. 1029/1999JA900025, 1999.

Scherliess, L., D. C. Thompson, and R. W. Schunk, Longitudinal variaibility of low-latitude total electron content: Tidal influences, J. Geophys. Res., 113, A01311, doi:10.1029/2007JA012480, 2008.

Tarpley, J. D., The ionospheric wind dynamo-II. Solar tides, Planet. Space Sci., 18, 1091-1103, 1970.

Thuillier, G., J. W. King, and A. J. Slater, An explanation of the longitudinal variation of the $\mathrm{O}^{1} \mathrm{D}(630 \mathrm{~nm})$ tropical nightglow intensity, J. Atmos. Terr. Phys., 38, 155-158, 1976.

Thuillier, G., R. H. Wiens, G. G. Shepherd, and R. G. Roble, Photochemistry and dynamics in thermospheric intertropical arcs measured by the WIND Imaging Interferometer on board UARS: A comparison with TIE-GCM simulations, J. Atmos. Terr. Phys., 64, 405-415, 2002.
VanZandt, T. E., W. L. Clark, and J. M. Warnock, Magnetic apex coordinates: a magnetic coordinate system for the ionospheric $F_{2}$ layer, $J$. Geophys. Res., 77(13), 2406-2411, 1972.

Walker, G. O., Longitudinal structure of the $F$-region equatorial anomaly-a review, J. Atmos. Terr. Phys., 43, 763-774, 1981.

Woodman, R., Vertical drift velocities and east-west electric fields at the magnetic equator, J. Geophys. Res., 75, 6249-6259, 1970.

Zhang, X., J. M. Forbes, M. E. Hagan, J. M. Russell, S. E. Palo, C. J. Mertens, and M. G. Mlynczak, Monthly tidal temperatures $20-120 \mathrm{~km}$ from TIMED/SABER, J. Geophys. Res., 111, A10S08, doi:10.1029/ 2005JA011504, 2006.

S. L. England (e-mail: england@ssl.berkeley.edu), X. Zhang, T. J. Immel, J. M. Forbes, and R. DeMajistre 